J. DIFFERENTIAL GEOMETRY

69 (2005) 43-74

\title{
COMPARISON THEOREM FOR KÄHLER MANIFOLDS AND POSITIVITY OF SPECTRUM
}

\author{
Peter Li \& Jiaping Wang
}

\begin{abstract}
The first part of this paper is devoted to proving a comparison theorem for Kähler manifolds with holomorphic bisectional curvature bounded from below. The model spaces being compared to are $\mathbb{C P}^{m}, \mathbb{C}^{m}$, and $\mathbb{C H}^{m}$. In particular, it follows that the bottom of the spectrum for the Laplacian is bounded from above by $m^{2}$ for a complete, $m$-dimensional, Kähler manifold with holomorphic bisectional curvature bounded from below by -1 . The second part of the paper is to show that if this upper bound is achieved and when $m=2$, then it must have at most four ends.
\end{abstract}

\section{Introduction}

In 1975, Cheng [1] proved a comparison theorem for the first Dirichlet eigenvalues of the Laplacian on geodesic balls. One of the consequences is a sharp upper bound for the bottom of the spectrum on a complete manifold with Ricci curvature bounded from below.

Theorem 0.1 (Cheng). Let $M^{n}$ be a complete Riemannian manifold of dimension $n$. Suppose the Ricci curvature of $M$ has a lower bound given by

$$
\operatorname{Ric}_{M} \geq-(n-1) \text {. }
$$

Then, the bottom of the spectrum of the Laplacian must satisfy the upper bound

$$
\lambda_{1}(M) \leq \frac{(n-1)^{2}}{4} .
$$

Cheng's estimate is sharp and equality is achieved by the hyperbolic space form $\mathbb{H}^{n}$. A key ingredient of Cheng's theorem is the Laplacian comparison theorem asserting that the Laplacian of the distance function $\Delta r$ has an upper bound for manifolds whose Ricci curvature is bounded from below.

Received 08/09/2004. 
A natural question is to study those manifolds satisfying the equality case in Cheng's theorem, i.e., $M$ satisfying

$$
\operatorname{Ric}_{M} \geq-(n-1)
$$

and

$$
\lambda_{1}(M)=\frac{(n-1)^{2}}{4} .
$$

Other than the fact that $\mathbb{H}^{n}$ is an example of the equality case, it was not known what can be said about this class of manifolds.

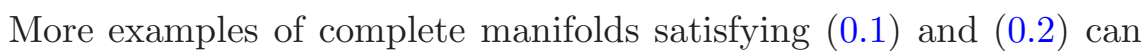
be found by considering hyperbolic manifolds $M=\mathbb{H}^{n} / \Gamma$ obtained by the quotient of $\mathbb{H}^{n}$ with a Kleinian group $\Gamma$. According to a theorem of Sullivan [1] the bottom of the spectrum, $\lambda_{1}(M)$, can be expressed by the Hausdorff dimension, $\delta(\Gamma)$, of the limit set of $\Gamma$. In fact, he proved that if $\Gamma$ is geometrically finite, then

$$
\lambda_{1}(M)=\left\{\begin{array}{lll}
\frac{(n-1)^{2}}{4}, & \text { if } & \delta(\Gamma) \leq \frac{n-1}{2} \\
\delta(\Gamma)(n-1-\delta(\Gamma)), & \text { if } & \delta(\Gamma) \geq \frac{n-1}{2} .
\end{array}\right.
$$

Hence, (0.2) is equivalent to $\delta(\Gamma) \leq \frac{n-1}{2}$ for geometrically finite $\Gamma$.

In 1995, Lee [6] proved that if $\bar{M}$ is a conformally compact Einstein manifold with

$$
\operatorname{Ric}_{M}=-(n-1),
$$

whose conformal infinity has non-negative Yamabe invariant, then ( is valid. This theorem provided more examples of manifolds satisfying

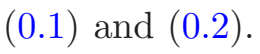

In [12], the authors proved the following theorems:

Theorem 0.2. Let $M^{n}$ be a complete Riemannian manifold of di-

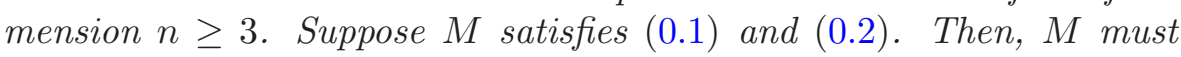
either be:

(1) A warped product manifold $M=\mathbb{R} \times N$ of dimension $n=3$ with metric given by

$$
d s_{M}^{2}=d t^{2}+\cosh ^{2} t d s_{N}^{2},
$$

where $N^{2}$ is a compact manifold with Gaussian curvature bounded from below by -1 ;

(2) A warped product manifold $M=\mathbb{R} \times N$ with metric given by

$$
d s_{M}^{2}=d t^{2}+e^{2 t} d s_{N}^{2},
$$

where $N^{n-1}$ is a compact manifold with non-negative Ricci curvature; or

(3) $M$ has only one end. 
When $n=2$, they proved that

Theorem 0.3. Let $M^{2}$ be a complete Riemannian surface. Suppose the Gaussian curvature of $M$ satisfies

$$
K_{M} \geq-1
$$

and

Then, $M$ must either be:

$$
\lambda_{1}(M)=\frac{1}{4}
$$

(1) A warped product manifold $M=\mathbb{R} \times \mathbb{S}^{1}$ with metric given by

$$
d s_{M}^{2}=d t^{2}+e^{2 t} d s_{\mathbb{S}^{1}},
$$

where $\mathbb{S}^{1}$ is the circle; or

(2) $M$ has no finite volume ends.

At this point, we should point out that in a previous work [11] of the authors where they generalized the theorems of Witten-Yau [i]

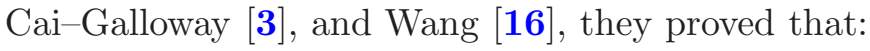

Theorem 0.4. Let $M^{n}$ be a complete Riemannian manifold of dimension $n \geq 3$. Suppose $M$ satisfies ( $\overline{0} \overline{i_{1}}$ ) and

$$
\lambda_{1}(M) \geq(n-2) .
$$

Then, $M$ must be either:

(1) A warped product manifold $M=\mathbb{R} \times N$ with metric given by

$$
d s_{M}^{2}=d t^{2}+\cosh (2 t) d s_{N}^{2},
$$

where $N^{n-1}$ is a compact manifold with Ricci curvature bounded from below by $-(n-2)$; or

(2) $M$ has only one end with infinite volume.

The purpose of this article is to investigate the corresponding setting for complete Kähler manifolds. The authors observed in [i] i i that on a Kähler manifold, one can rule out the existence of two infinite volume ends much easier than the Riemannian case, hence prompted this study. A major new ingredient in this paper is a comparison theorem (Theo-

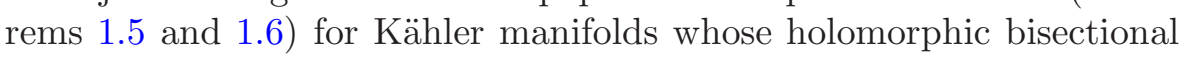
curvature is bounded from below. It is a general principle that holomorphic bisectional curvature is more suitable for the Kähler category. Though assumptions on the holomorphic bisectional curvature are more restrictive compared to assumptions on the Ricci curvature, the results obtained, however, should be sharper.

Now, let us assume that $M^{m}$ is a Kähler manifold of complex dimension $m$. Let $\left\{e_{1}, \cdots, e_{m}\right\}$ be a unitary frame for the $(1,0)$-part of 
the complexified tangent space, $T_{x}^{1,0} M$. The holomorphic bisectional curvature is denoted by

$$
R_{\alpha \bar{\alpha} \beta \bar{\beta}}=\left\langle R_{e_{\alpha} e_{\bar{\alpha}}} e_{\beta}, e_{\bar{\beta}}\right\rangle
$$

for $\alpha, \beta=1, \cdots, m$.

Definition 0.5. Let $M^{m}$ be a Kähler manifold of complex dimension $m$. We say that the holomorphic bisectional curvature of $M$ is bounded from below by a constant $K$, denoted by

$$
B K_{M} \geq K
$$

if

$$
R_{\alpha \bar{\alpha} \beta \bar{\beta}} \geq K\left(1+\delta_{\alpha \beta}\right)
$$

for any unitary frame $\left\{e_{1}, e_{2}, \ldots, e_{m}\right\}$.

Note that for the simply connected complex space forms $\mathbb{C P}^{n}, \mathbb{C}^{n}$ and $\mathbb{C} \mathbb{H}^{n}$, their holomorphic bisectional curvatures satisfy

$$
R_{\alpha \bar{\alpha} \beta \bar{\beta}}=K\left(1+\delta_{\alpha \beta}\right),
$$

where $K=1,0$ and -1 , respectively.

We would like to point out that a complex Hessian comparison theorem for the Busemann function was proved by Greene-Wu ["ris] in 1978 for Kähler manifolds with non-negative holomorphic bisectional curvature, i.e., $B K_{M} \geq 0$. In their recent paper 4 ], Cao-Ni proved the complex Hessian comparison theorem for the distance function on a Kähler manifold with $B K_{M} \geq 0$. Since the assumption $B K_{M} \geq-1$ is not the same for the cases $\alpha=\beta$ and $\alpha \neq \beta$, it is difficult to come up with a comparison theorem. In Section 1, we gave a new proof of the Hessian comparison theorem for the Riemannian case which allows us to generalize to the Kähler case.

A consequence of the comparison theorem (Theorem is a version of Cheng's upper bound for $\lambda_{1}(M)$ for Kähler manifolds with $B K_{M} \geq-1$. In fact, we proved (Corollary $\left.1 . \overline{1} \overline{1}_{1}\right)$ that

$$
\lambda_{1}(M) \leq m^{2} .
$$

Similar to Cheng's estimate, this estimate is also sharp as equality is achieved by the complex hyperbolic space form $\mathbb{C H} \mathbb{H}^{m}$. Of course, one now faces the question of what can be said about those Kähler manifolds satisfying

$$
B K_{M} \geq-1
$$

and

$$
\lambda_{1}(M)=m^{2} .
$$




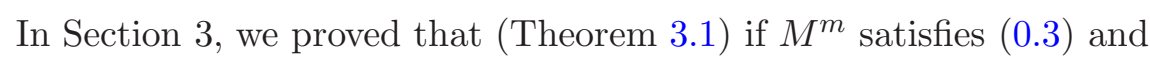

$$
\lambda_{1}(M) \geq m,
$$

then $M$ must have only one end with infinite volume. In particular, for those manifolds satisfying $\left(\overline{0} \cdot \overline{3}_{i}^{\prime}\right)$ and $\left(\overline{0} \cdot \overline{1}^{\prime}\right)$, we will only have to content with finite volume ends.

Finally, in Section 4, we considered complete Kähler surfaces satisfying $\left(\bar{D} . \overline{3}^{i}\right)$ and $\left(\overline{0} \cdot \overline{4}^{3}\right)$. We showed that such a surface must have at most 4 ends, one of which has infinite volume and the rest have finite volumes. Unfortunately, we do not know if this is sharp, and we suspect that it is not. We also suspect that this finiteness phenomenon should also be true in high dimensions.

\section{Comparison theorems}

In this section, we will prove a sharp comparison theorem for Kähler manifolds satisfying curvature bounds. We will start by giving a new proof for the Riemannian case to illustrate the ideas. This argument, which relies on the commutation formula for covariant derivatives, also gives a slight extension of the Riemannian case.

Let $\left(M^{n}, g\right)$ be a complete Riemannian manifold and let $r(x)=$ $d(x, p)$ be the distance function to a fixed point $p \in M$. For any unit vector $V$ in the unit tangent sphere $S_{p}^{n-1}(M)$, we define

$$
\rho(V)=\sup \left\{T: \gamma_{V}(t)=\exp _{p}(t V) \text { is minimizing on }[0, T]\right\}
$$

to be the maximum distance for the geodesic in the direction of $V$ to be minimizing. We also let

$$
C_{p}=\left\{\rho(V) V: \rho(V)<\infty, V \in S_{p}^{n-1}(M)\right\}
$$

to be the tangential cut locus of $p$. The cut locus of $p \in M$ is denoted by $\operatorname{Cut}(p)=\exp _{p}\left(C_{p}\right)$. Moreover,

$$
M=\exp _{p}(\Sigma(p)) \cup \operatorname{Cut}(p),
$$

where

$$
\Sigma(p)=\left\{t V: 0 \leq t<\rho(V), V \in S_{p}^{n-1}(M)\right\}
$$

and

$$
\exp _{p}: \Sigma(p) \rightarrow \exp _{p}(\Sigma(p))
$$

is a diffeomorphism. It is known that the set $\operatorname{Cut}(p)$ has measure zero in $M$. The polar coordinate system $(r, \theta)$ on the tangent space $T_{p}(M)$ also induces a coordinate chart on $\exp _{p}(\Sigma(p))$. The definition of exponential map implies that $r(x)=t$ if $x=\exp _{p}(t \theta)$ for $t<\rho(\theta)$. Moreover, $r(x)$ is smooth on $\exp _{p}(\Sigma(p)) \backslash\{p\}$ and $|\nabla r|=1$ on $\exp _{p}(\Sigma(p)) \backslash\{p\}$.

We begin by defining the following notion of curvature. 
Definition 1.1. For any integer $1 \leq \ell \leq n-1$, we defined the $\ell$ sectional curvature of a pair $\{w, V\}$, where $w \in T_{p} M$ and $V \subset T_{p} M$ is an $\ell$-dimensional subspace perpendicular to $w$, by

$$
K_{M}^{\ell}(w, V)=\sum_{i=1}^{\ell}\left\langle R_{w e_{i}} w, e_{i}\right\rangle
$$

with $\left\{e_{1}, e_{2}, \ldots, e_{\ell}\right\}$ being an orthonormal basis for $V$.

Note that $K_{M}^{\ell}(w, V)$ does not depend on the choice of orthonormal basis $\left\{e_{i}\right\}$. We say that a manifold $M$ has $\ell$-sectional curvature bounded from below by a constant $K$ if

$$
K_{M}^{\ell}(w, V) \geq \ell K
$$

for all pairs $\{w, V\}$ at any point $p \in M$. When $\ell=1$, this is equivalent to saying that the sectional curvature $K_{M} \geq K$. When $\ell=n-1$, this is equivalent to the Ricci curvature bounded by

$$
\operatorname{Ric}_{M} \geq(n-1) K \text {. }
$$

To set up our model for the comparison theorem, we consider $M_{K}^{\ell+1}$ to be the $(\ell+1)$-dimensional, simply connected, space form of constant sectional curvature $K$. For a fixed origin $\bar{p} \in M_{K}^{\ell+1}$, we denote the distance function from any point $\bar{x}$ to $\bar{p}$ by $\bar{r}(\bar{x})$.

Theorem 1.2. Let $M$ be a complete Riemannian manifold of dimension $n$. Assume that the $\ell$-sectional curvatures of $M$ satisfy $K_{M}^{\ell} \geq \ell K$. Then, within the cut locus of a fixed point $p \in M$ and for any $V \subset T_{x} M$ perpendicular to $\nabla r(x)$,

$$
\sum_{i=1}^{\ell} D^{2}(r)\left(e_{i}, e_{i}\right) \leq \sum_{i=1}^{\ell} \bar{D}^{2}(\bar{r})\left(\bar{e}_{i}, \bar{e}_{i}\right)
$$

with $\left\{e_{1}, \ldots e_{\ell}\right\}$ being any orthonormal basis of $V$ and $\left\{\bar{e}_{1}, \ldots \bar{e}_{\ell}\right\}$ being an orthonormal basis of $T_{\bar{p}} M_{K}^{\ell+1}$ with $\bar{e}_{i} \perp \bar{\nabla} r$.

Proof. For $x \in \exp _{p}(\Sigma(p)) \backslash\{p\}$, let $\gamma$ be the minimal normal geodesic joining $p$ to $x$. At $x$, we choose an orthonormal frame $\left\{e_{1}, \ldots, e_{n}\right\}$, such that $e_{1}=\nabla r$. By parallel translating the frame $\left\{e_{i}\right\}$, we obtain an orthonormal frame along $\gamma$ also denoted by $\left\{e_{i}\right\}_{i=1}^{n}$ with the property that $e_{1}=\nabla r$. Since $|\nabla r|^{2}=1$ on $\exp _{p}(\Sigma(p)) \backslash\{p\}$, by taking covariant derivative of this equation, we obtain

$$
\begin{aligned}
0 & =\left(|\nabla r|^{2}\right)_{\alpha \alpha} \\
& =2 \sum_{i=1}^{n} r_{i \alpha} r_{i \alpha}+2 \sum_{i=1}^{n} r_{i} r_{i \alpha \alpha}
\end{aligned}
$$


for each $2 \leq \alpha \leq n$. Since $\gamma$ is a geodesic and each $e_{i}$ is parallel along $\gamma$, each term on the right-hand side of (i. covariant derivatives. The commutation formula for covariant derivative then implies

$$
\sum_{i=1}^{n} r_{i} r_{i \alpha \alpha}=\sum_{i=1}^{n} r_{i} r_{\alpha \alpha i}+\sum_{i, j=1}^{n} R_{i \alpha j \alpha} r_{i} r_{j}
$$

Substituting into $(1,1)$ and using the fact that $|\nabla r|=1=r_{1}$, we obtain

$$
0 \geq 2 r_{\alpha \alpha}^{2}+2 \frac{\partial\left(r_{\alpha \alpha}\right)}{\partial r}+2 K_{M}\left(e_{1}, e_{\alpha}\right)
$$

Suppose $V \subset T_{x} M$ is spanned by $\left\{e_{2}, \ldots, e_{\ell+1}\right\}$, then summing over $\alpha=2, \ldots, \ell+1,(1.2)$ becomes

$$
0 \geq \sum_{\alpha=2}^{\ell+1} r_{\alpha \alpha}^{2}+\frac{\partial}{\partial r}\left(\sum_{\alpha=2}^{\ell+1} r_{\alpha \alpha}\right)+K_{M}^{\ell}\left(e_{1}, V\right) .
$$

Using the lower bound of the $\ell$-sectional curvature, the inequality

$$
\sum_{\alpha=2}^{\ell+1} r_{\alpha \alpha}^{2} \geq \frac{1}{\ell}\left(\sum_{\alpha=2}^{\ell+1} r_{\alpha \alpha}\right)^{2}
$$

and by setting $f(t)=\sum_{\alpha=2}^{\ell+1} r_{\alpha \alpha}(\gamma(t))$, (1) $\overline{3}^{1}$ in can be expressed as

$$
0 \geq \frac{1}{\ell} f^{2}(t)+f^{\prime}(t)+\ell K .
$$

Note that since a smooth Riemannian metric is locally Euclidean,

$$
\lim _{t \rightarrow 0} t f(t)=\ell .
$$

We will now consider the three separate cases when $K=0, K>0$, and $K<0$.

Case 1. When $K=0$, inequality (1.4) becomes

$$
f^{\prime}(t)+\frac{1}{\ell} f^{2}(t) \leq 0
$$

This implies that $f^{\prime}(t) \leq 0$ and $f(t)$ is a decreasing function. Let $(0, T)$ be the largest interval such that $f(t)>0$, then we have

$$
\left(\frac{1}{f}\right)^{\prime}=-\frac{f^{\prime}}{f^{2}} \geq \frac{1}{\ell}
$$

and $f(t) \leq \frac{\ell}{t}$ on $(0, T)$. Since $f(t) \leq 0$ for $t \geq T$, we can still conclude that $f(t) \leq \frac{\ell}{t}$ on $(0, \rho(\theta))$. 
Case 2. When $K>0$, inequality (1.4) can be written as

$$
\frac{\ell f^{\prime}(t)}{f^{2}(t)+\ell^{2} K} \leq-1 \text {. }
$$

This implies that

$$
\frac{d}{d t} \tan ^{-1}\left(\frac{f}{\ell \sqrt{K}}\right) \leq-\sqrt{K}
$$

Integrating from 0 to $t$, we have

$$
\tan ^{-1}\left(\frac{f}{\ell \sqrt{K}}\right) \leq \frac{\pi}{2}-\sqrt{K} t
$$

implying that

$$
f(t) \leq \ell \sqrt{K} \cot (\sqrt{K} t) .
$$

Case 3. When $K<0$, let $T$ be the first time such that

$$
f^{2}(t)+\ell^{2} K=0
$$

Then, on $(0, T)$, we have $f^{2}(t)+\ell^{2} K>0$ and

$$
\frac{\ell f^{\prime}(t)}{f^{2}(t)+\ell^{2} K} \leq-1 \text {. }
$$

This implies that

$$
\frac{d}{d t} \operatorname{coth}^{-1}\left(\frac{f}{\ell \sqrt{|K|}}\right) \geq \sqrt{|K|}
$$

and

$$
f(t) \leq \ell \sqrt{|K|} \operatorname{coth}(\sqrt{|K|} t)
$$

on $(0, T)$. For $t \geq T$, we claim that $f(t) \leq \ell \sqrt{|K|}$. Indeed, if $f\left(t_{1}\right)>$ $\ell \sqrt{|K|}$ for $t_{1}>T$, then there exists $t_{2} \in\left(T, t_{1}\right)$ such that $f^{\prime}\left(t_{2}\right) \geq 0$ and $f\left(t_{2}\right)>\ell \sqrt{|K|}$. In this case,

$$
f^{\prime}\left(t_{2}\right)+\frac{1}{\ell} f^{2}\left(t_{2}\right)+\ell K>0
$$

which is a contradiction. Thus,

$$
f(t) \leq \ell \sqrt{|K|}
$$

for $T \leq t<\rho(\theta)$, and we conclude that

$$
f(t) \leq \ell \sqrt{|K|} \operatorname{coth}(\sqrt{|K|} t)
$$

for $0<t<\rho(\theta)$.

The Theorem follows by observing that $r_{11}=0$ and that the above inequalities become equalities on a simply connected space form with constant sectional curvature.

q.e.d. 
Observe that the standard Laplacian comparison theorem and the Hessian comparison theorem follow from Theorem 1.2 by setting $\ell=n-1$ and $\ell=1$, respectively. Moreover, the Bishop comparison theorem is also a corollary. Indeed, if we consider the polar coordinate system $(r, \theta)$, Gauss lemma implies that

$$
d s_{M}^{2}=d r^{2}+g_{\alpha \beta}(r, \theta) d \theta^{\alpha} d \theta^{\beta} \quad, \alpha, \beta=2, \ldots, n .
$$

If we denote

$$
J(r, \theta)=\sqrt{\operatorname{det}\left(g_{\alpha \beta}\right)}
$$

to be the area element of the geodesic sphere $\partial B_{p}(r)$, then

$$
\Delta_{M}=\frac{\partial^{2}}{\partial r^{2}}+\frac{\partial}{\partial r}(\ln J) \frac{\partial}{\partial r}+\Delta_{\partial B_{p}(r)} .
$$

Thus,

$$
\Delta_{M} r=\frac{\partial}{\partial r}(\ln J)
$$

on $\exp _{p}(\Sigma(p)) \backslash\{p\}$.

Corollary 1.3 (Bishop). If $\operatorname{Ric}_{M} \geq(n-1) K$, then

$$
\frac{J(r, \theta)}{J_{K}(r)}
$$

is a non-increasing function of $r$, where $J_{K}(r)$ is the area element of the geodesic sphere of radius $r$ in the space form $M_{K}^{n}$ given by

$$
J_{K}(r)=\left\{\begin{array}{lll}
\sin ^{n-1}(\sqrt{K} r), & \text { if } & K>0 \\
r^{n-1}, & \text { if } & K=0 \\
\sinh ^{n-1}(\sqrt{|K|} r), & \text { if } & K<0 .
\end{array}\right.
$$

Moreover, if $A_{p}(r)$ and $V_{p}(r)$ denote the area of $\partial B_{p}(r)$ and the volume of $B_{p}(r)$, respectively, then

$$
\frac{A_{p}\left(r_{2}\right)}{A_{p}\left(r_{1}\right)} \leq \frac{J_{K}\left(r_{2}\right)}{J_{K}\left(r_{1}\right)}
$$

and

$$
\frac{V_{p}\left(r_{2}\right)}{V_{p}\left(r_{1}\right)} \leq \frac{\int_{0}^{r_{2}} J_{K}(r) d r}{\int_{0}^{r_{1}} J_{K}(r) d r}
$$

for $r_{1} \leq r_{2}$.

The following theorem is a global version of the Laplacian comparison theorem. For a proof, we refer to [7] 
Corollary 1.4. If $\operatorname{Ric}_{M} \geq(n-1) K$, then

$$
\Delta r(x) \leq \bar{\Delta} \bar{r}(r(x))
$$

in the sense of distributions, where $\bar{\Delta}$ is the Laplacian on the space form $M_{K}^{n}$ and $\bar{r}$ is the distance function of $M_{K}^{n}$ with respect to a fixed point. That is to say, for any $\varphi \in C_{0}^{\infty}(M)$ with $\varphi \geq 0$, we have

$$
\int_{M} r(x) \Delta \varphi(x) \leq \int_{M}(\bar{\Delta} \bar{r}) \varphi .
$$

We are now ready to prove the comparison for Kähler manifolds. Recall that if the Kähler metric of $M$ is given by $d s^{2}=h_{\alpha \bar{\beta}} d z^{\alpha} d \bar{z}^{\beta}$, then the gradient and the Laplacian is given by

$$
\langle\nabla f, \nabla g\rangle=2 h^{\alpha \bar{\beta}}\left(f_{\alpha} g_{\bar{\beta}}+f_{\bar{\beta}} g_{\alpha}\right)
$$

and

$$
\Delta f=4 h^{\alpha \bar{\beta}} \frac{\partial^{2} f}{\partial z^{\alpha} \partial \bar{z}^{\beta}} .
$$

Theorem 1.5. Let $M^{m}$ be a complete Kähler manifold such that its bisectional curvature $B K_{M} \geq 0$. Then, on $\exp _{p}(\Sigma(p)) \backslash\{p\}$, we have

$$
\left(r^{2}\right)_{\alpha \bar{\alpha}} \leq 1 \text {. }
$$

Proof. For any $x \in M$, we choose a unitary frame $\left\{e_{1}, \cdots, e_{m}\right\}$ at $x$ and parallel translate each $e_{\alpha}$ along the minimizing geodesic $\gamma$. We also parallel translate each $e_{\alpha}$ so that they are defined on a neighborhood of $\gamma$. Setting $u=r^{2}$, a similar calculation as in the Riemannian case above shows that

$$
\begin{aligned}
|\partial u|_{\alpha \bar{\alpha}}^{2}= & \sum_{\delta}\left(u_{\delta} u_{\bar{\delta}}\right)_{\alpha \bar{\alpha}} \\
= & \sum_{\delta}\left(\left|u_{\delta \bar{\alpha}}\right|^{2}+\left|u_{\alpha \delta}\right|^{2}\right)+u_{\alpha \bar{\alpha} \delta} u_{\bar{\delta}}+u_{\alpha \bar{\alpha} \bar{\delta}} u_{\delta}+R_{\alpha \bar{\alpha} \delta \bar{\eta}} u_{\bar{\delta}} u_{\eta} \\
& \geq \frac{1}{2}\left\langle\nabla u_{\alpha \bar{\alpha}}, \nabla u\right\rangle+\left|u_{\alpha \bar{\alpha}}\right|^{2} .
\end{aligned}
$$

Let $f(t)=u_{\alpha \bar{\alpha}}(\gamma(t))$. Then, we have

$$
f^{2}(t)+t f^{\prime}(t) \leq f(t)
$$

and $f(0)=1$. If there exists $t>0$ such that $f(t) \geq 1+\epsilon$ for some $\epsilon>0$, then using the initial condition

$$
\lim _{r \rightarrow 0}\left(r^{2}\right)_{\alpha \bar{\alpha}}=1
$$

there must be a $0<t_{1} \leq t$ such that $f^{\prime}\left(t_{1}\right) \geq 0$ and $f\left(t_{1}\right)=1+\epsilon$. This contradicts the differential inequality above and the theorem follows.

q.e.d. 
Theorem 1.6. Let $M^{m}$ be a complete Kähler manifold with its holomorphic bisectional curvature satisfying the bound $B K_{M} \geq-1$. Then, on $\exp _{p}(\Sigma(p)) \backslash\{p\}$, we have

$$
\begin{aligned}
\Delta r(x) & \leq 2(m-1) \operatorname{coth}(r(x))+2 \operatorname{coth}(2 r(x)) \\
& =\bar{\Delta} \bar{r}(r(x)),
\end{aligned}
$$

where $\bar{\Delta}$ and $\bar{r}$ are the Laplacian and the distance function of the model manifold $\mathbb{C H}^{m}$.

Proof. For any $x$, we choose a unitary frame $\left\{e_{1}, \cdots, e_{m}\right\}$ at point $x$ such that

$$
e_{1}=\frac{1}{2}(\nabla r-\sqrt{-1} J \nabla r)
$$

We parallel translate each $e_{\alpha}$ along the minimizing geodesic $\gamma$ between $p$ and $x$ and then to a neighborhood of $\gamma$. Along $\gamma$, one easily checks that the Hessian of $r$ must satisfy $r_{11}=-r_{1 \overline{1}}$. Therefore,

$$
\begin{aligned}
0 & =|\nabla r|_{1 \overline{1}}^{2} \\
& =2\left|r_{1 \overline{1}}\right|^{2}+2\left|r_{11}\right|^{2}+\left\langle\nabla r_{1 \overline{1}}, \nabla r\right\rangle+2 R_{1 \overline{1} 1 \overline{1}} r_{1} r_{\overline{1}} \\
& \geq 4\left|r_{1 \overline{1}}\right|^{2}+\frac{\partial}{\partial r}\left(r_{1 \overline{1}}\right)-1 .
\end{aligned}
$$

Let $f(t)=r_{1 \overline{1}}(\gamma(t))$. Then, we have

$$
4 f^{2}(t)+f^{\prime}(t) \leq 1
$$

and $\lim _{t \rightarrow 0} t f(t)=\frac{1}{4}$. It is then not difficult to see that

$$
f(t) \leq \frac{1}{2} \operatorname{coth}(2 t)
$$

For $\alpha \neq 1$, we have

$$
\begin{aligned}
0 & =|\nabla r|_{\alpha \bar{\alpha}}^{2} \\
& \geq 2\left|r_{\alpha \bar{\alpha}}\right|^{2}+\left\langle\nabla r_{\alpha \bar{\alpha}}, \nabla r\right\rangle+2 R_{1 \overline{1} \alpha \bar{\alpha}} r_{1} r_{\overline{1}} \\
& \geq 2\left|r_{\alpha \bar{\alpha}}\right|^{2}+\frac{\partial}{\partial r}\left(r_{\alpha \bar{\alpha}}\right)-\frac{1}{2} .
\end{aligned}
$$

Let $w(t)=r_{\alpha \bar{\alpha}}(\gamma(t))$. Then, we have

$$
4 w^{2}(t)+2 w^{\prime}(t) \leq \frac{1}{2}
$$

and $\lim _{t \rightarrow 0} t w(t)=1$, hence

$$
w(t) \leq \frac{1}{2} \operatorname{coth}(t) .
$$


Finally, we have

$$
\begin{aligned}
\Delta r & =4 \sum_{\alpha=1}^{m} r_{\alpha \bar{\alpha}} \\
& \leq 2(m-1) \operatorname{coth}(r)+2 \operatorname{coth}(2 r) .
\end{aligned}
$$

Equivalently, this can be written as

$$
\Delta(\ln (\cosh (r))) \geq 2 m .
$$

One computes readily that equality is achieved on $\mathbb{C} \mathbb{H}^{m}$.

q.e.d.

Corollary 1.7. Let $M^{m}$ be a complete Kähler manifold with its holomorphic bisectional curvature satisfying the bound $B K_{M} \geq-1$. Then, for any $x \in M$ and $0 \leq r \leq R$, the volume of the geodesic balls satisfy

$$
\frac{V_{x}(R)}{V_{x}(r)} \leq \frac{V_{\mathbb{C H}^{m}}(R)}{V_{\mathbb{C H}^{m}}(r)},
$$

where $V_{\mathbb{C H}^{m}}(r)$ denotes the volume of the geodesic ball of radius $r$ in $\mathbb{C H}^{m}$. In particular, the bottom of the spectrum of $M$ has an upper bound given by $\lambda_{1}(M) \leq m^{2}$.

Proof. The volume comparison theorem follows similar to the Riemannian case by applying Theorem 1.6. Taking $r=1$ in the volume comparison inequality, we have

$$
\begin{aligned}
V_{p}(R) & \leq C V_{\mathbb{C H}^{m}}(R) \\
& \leq C e^{2 m R}
\end{aligned}
$$

for all $R \geq 1$. However, in [i.

$$
V_{p}(R) \geq C \exp \left(2 \sqrt{\lambda_{1}(M)} R\right) .
$$

Combining with the upper bound, we conclude that $\lambda_{1}(M) \leq m^{2}$ as claimed.

q.e.d.

Theorem 1.8. Let $M^{m}$ be a complete Kähler manifold with its holomorphic bisectional curvature satisfying the bound $B K_{M} \geq 1$. Then, on $\exp _{p}(\Sigma(p)) \backslash\{p\}$, we have

$$
\begin{aligned}
\Delta r(x) & \leq 2(m-1) \cot (r(x))+2 \cot (2 r(x)) \\
& =\bar{\Delta} \bar{r}(r(x)),
\end{aligned}
$$

where $\bar{\Delta}$ and $\bar{r}$ are the Laplacian and the distance function of the model manifold $\mathbb{C P}^{m}$. 
Proof. Following the argument as in the proof of Theorem i1.6, except (1.5) and (1.6) become

$$
4 f^{2}(t)+f^{\prime}(t) \leq-1
$$

and

$$
f(t) \leq \frac{1}{2} \cot (2 t)
$$

respectively. Also (1.7) and (1.8) become

$$
4 w^{2}(t)+2 w^{\prime}(t) \leq-\frac{1}{2}
$$

and

$$
w(t) \leq \frac{1}{2} \cot (t)
$$

respectively. The theorem now follows as claimed.

q.e.d.

Corollary 1.9. Let $M^{m}$ be a complete Kähler manifold with $B K_{M} \geq$ 1. Then, the diameter $d(M)$ of $M$ is bounded above by

$$
d(M) \leq \frac{\pi}{2}
$$

which is the diameter of the model space $\mathbb{C P}^{m}$. Moreover, the volume of $M$ is bounded by

$$
\begin{aligned}
V(M) & \leq \frac{1}{m} \sin ^{2 m}(d(M)) \\
& \leq V\left(\mathbb{C P}^{m}\right) .
\end{aligned}
$$

Proof. Suppose the diameter of $M$ is greater than $\frac{\pi}{2}$. Then, there exists a pair of points $p, x \in M$ such that $r(x)>\frac{\pi}{2}$ and $x \in \exp _{p}(\Sigma(p)) \backslash$ $\{p\}$. Using the fact that $\Delta r(x)$ is given by the mean curvature $H(x)$ of the geodesic sphere of radius $r(x)$ at $x$, the bound given by Theorem 1.8 asserts that the function $r$ cannot be smooth since the upper bound

$$
2(m-1) \cot (r)+2 \cot (2 r)
$$

becomes $-\infty$ at $r=\frac{\pi}{2}$. This contradicts the assumption that $d(M)>\frac{\pi}{2}$, and the first part of the theorem follows.

If we write $x=(r, \theta)$ in polar coordinates and let $A(r, \theta)$ be the area element of the sphere of radius $r$ centered at $p$, then $\frac{\partial}{\partial r} A(r, \theta)=$ $H(r, \theta) A(r, \theta)$. The comparison theorem then asserts that

$$
A^{-1}(t, \theta) \frac{\partial A(t, \theta)}{\partial r} \leq 2(m-1) \cot (t)+2 \cot (2 t) .
$$


Integrating over the interval $0 \leq t \leq r$, we obtain

$$
\begin{aligned}
A(r, \theta) & \leq \sin ^{2(m-1)} r \sin 2 r \\
& =2 \sin ^{2 m-1} r \cos r .
\end{aligned}
$$

Following the same argument as in the Riemannian case (see [i]i]), we conclude the volume comparison

$$
\begin{aligned}
V(M) & =V_{p}(d(M)) \\
& \leq 2 \int_{0}^{d(M)} \sin ^{2 m-1} r \cos r d r \\
& =\frac{1}{m} \sin ^{2 m}(d(M)) .
\end{aligned}
$$

q.e.d.

Following Cheng's argument [i]i], one can also conclude the following eigenvalue comparison theorem.

Corollary 1.10. Let $M^{m}$ be a complete Kähler manifold with holomorphic bisectional curvature bounded from below by $K$, where $K$ is either 1,0 , or -1 . Then, the first Dirichlet eigenvalue, $\lambda_{1}\left(B_{p}(r)\right)$, of the geodesic ball of radius $r$ centered at $p \in M$ must be bounded from above by

$$
\lambda_{1}\left(B_{p}(r)\right) \leq \lambda_{1}\left(B_{\bar{M}}(r)\right),
$$

where $\lambda_{1}\left(B_{\bar{M}}(r)\right)$ is the first Dirichlet eigenvalue of the geodesic ball of radius $r$ on the model manifold $\bar{M}^{m}$. The model is taken to be $\mathbb{C P}^{m}$, $\mathbb{C}^{m}$, or $\mathbb{C H}^{m}$ for $K$ being 1 , 0 , or -1 , respectively.

\section{Estimates for harmonic functions}

Throughout this section, we assume $M^{m}$ is a complete Kähler manifold of complex dimension $m$ with holomorphic bisectional curvature bounded by

$$
B K_{M} \geq-1 \text {. }
$$

We also assume that the bottom spectrum of $M$ satisfies

$$
\lambda_{1}(M) \geq m^{2} .
$$

The first step is to give precise estimates on the volume growth or volume decay of an end of $M$. The volume estimates will then be used to derive sharp estimates for the barrier harmonic functions on the corresponding end.

Recall that an end $E$ is defined to be an unbounded component of $M \backslash D$ for some compact set $D$. Without loss of generality, we may 
assume that $D=B_{p}\left(R_{0}\right)$ is a geodesic ball centered at some fixed point $p \in M$ with radius $R_{0}>0$. We will denote $V_{E}(R)$ to be the volume of the set $B_{p}(R) \cap E$, and $V_{E}(\infty)$ is simply the volume of $E$. Also, we recall (see [8] and [9.9-1) that an end $E$ is said to be a non-parabolic (or parabolic) end if it admits (or does not admit) a positive Green's function for the Laplacian on $E$ with Neumann boundary condition on $\partial E$.

Let us first recall Theorem 1.4 of [11] 1 in stated for the class of manifolds being considered.

Theorem 2.1. Let $E$ be an end of a complete Kähler manifold $M$ satifying (2.2). Then, either

(1) $E$ is a parabolic end with finite volume, and it must have exponential volume decay given by

$$
V_{E}(\infty)-V_{E}(R) \leq C_{1} \exp (-2 m R)
$$

for $R \geq R_{0}+1$ and some constant $C_{1}>0$ depending only on $E$; or

(2) $E$ is a non-parabolic end with infinite volume, and it must have exponential volume growth given by

$$
V_{E}(R) \geq C_{2} \exp (2 m R)
$$

for $R \geq R_{0}+1$ and some constant $C_{2}>0$ depending only on $E$.

On the other hand, if $M$ satisfies (2.1), then by setting $r=1$ in Corollary 1 , we conclude that for any $x \in M$,

$$
V_{p}(R) \leq C_{3} \exp (2 m R)
$$

for sufficiently large $R$. On the other hand, if we let $x \in \partial B_{p}\left(R_{1}\right), r=1$ and $R=R_{1}+1$ in Corollary $\overline{1} \overline{1}$, then we have

$$
\begin{aligned}
V_{x}(1) & \geq C_{4} V_{x}\left(R_{1}+1\right) \exp \left(-2 m\left(R_{1}+1\right)\right) \\
& \geq C_{4} V_{p}(1) \exp \left(-2 m\left(R_{1}+1\right)\right) .
\end{aligned}
$$

Since $B_{x}(1) \subset B_{p}\left(R_{1}+1\right)$, this can be rewritten as

$$
V_{p}(R) \geq C_{5} \exp (-2 m R)
$$

for $x \in \partial B_{p}(R)$. Combining (2.3), (2.4) with Theorem $\overline{2} . \overline{1}$, we obtain the following corollary.

Corollary 2.2. Let $M^{m}$ be a complete Kähler manifold satisfying (2.1) and (2.2). Let $p \in M$ be a fixed point and $E$ be an end of $M$ given by an unbounded component of $M \backslash B_{p}\left(R_{0}\right)$. Then, either

(1) $E$ is a parabolic end with finite volume, and it must have exponential volume decay given by

$$
C_{1} \exp (-2 m R) \leq V_{E}(\infty)-V_{E}(R) \leq C_{2} \exp (-2 m R)
$$


for $R \geq R_{0}+1$ and some constants $0<C_{1}<C_{2}$ depending only on $E$; or

(2) $E$ is a non-parabolic end with infinite volume, and it must have exponential volume growth given by

$$
C_{3} \exp (2 m R) \leq V_{E}(R) \leq C_{4} \exp (2 m R)
$$

for $R \geq R_{0}+1$ and some constants $0<C_{3} \leq C_{4}$ depending only on $E$.

According to Theorem 0.1 in [11], the condition $\lambda_{1}(M)>0$ implies that $M$ must have infinite volume. Hence, we may assume that $M$ has a non-parabolic end $E_{1}$. In the following discussion, we assume that $M$ also has a finite volume, parabolic end $E_{2}$.

Recall that the theory of Li-Tam [9] (also see [i8 exists a positive harmonic function $f$ satisfying the following properties:

(1) $\inf _{\partial B_{p}(r) \cap E_{1}} f(x) \rightarrow 0$ as $r \rightarrow \infty$;

(2) $\sup _{\partial B_{p}(r) \cap E_{2}} f(x) \rightarrow \infty$ as $r \rightarrow \infty$; and

(3) $f$ is bounded and has finite Dirichlet integral on $M \backslash E_{2}$.

In order to obtain the appropriate estimates on $f$, we will give an outline of the construction. Let us consider the sequence of harmonic functions $v_{R}$ satisfying

$$
\begin{array}{rll}
\Delta v_{R}=0 & \text { on } & E_{1}(R), \\
v_{R}=1 & \text { on } & \partial E_{1},
\end{array}
$$

and

$$
v_{R}=0 \quad \text { on } \quad \partial B_{p}(R) \cap E_{1} .
$$

The assumption that $E_{1}$ is non-parabolic implies that $v_{R}$ converges uniformly on compact subsets of $E_{1}$ to a non-constant harmonic function $v$.

Similarly, let $u_{R}$ be a sequence of harmonic functions satisfying

$$
\begin{array}{rll}
\Delta u_{R}=0 & \text { on } & E_{2}(R), \\
u_{R}=0 & \text { on } & \partial E_{2},
\end{array}
$$

and

$$
u_{R}=c_{R} \quad \text { on } \quad \partial B_{p}(R) \cap E_{2} .
$$

The assumption that $E_{2}$ is parabolic implies that there exist a subsequence $R_{i} \rightarrow \infty$ and a sequence of constants $c_{i}=c_{R_{i}} \rightarrow \infty$ such that the sequence of functions

$$
u_{i}=u_{R_{i}}
$$

converges uniformly on compact subsets of $E_{2}$ to a harmonic function $u$. Multiplying $u$ by a constant if necessary, we may assume that

$$
\int_{\partial E_{1}} \frac{\partial v}{\partial \nu}=-\int_{\partial E_{2}} \frac{\partial u}{\partial \nu}
$$


After this normalization, it was proved (also see [15] that there exists a harmonic function $f$ defined on $M$ which is bounded distance from $v$ and $u$ on the corresponding ends $E_{1}$ and $E_{2}$. Moreover, $f$ will satisfy the properties stated above.

It was proved in Lemma 1.2 of [i] i i that on $M \backslash E_{2}$, the Dirichlet integral of the function $f$ must satisfy the decay estimate

$$
\int_{\left(B_{p}(R+1) \backslash B_{p}(R)\right) \backslash E_{2}}|\nabla f|^{2} \leq C \exp (-2 m R)
$$

for $R$ sufficiently large.

Theorem 2.3. Let $M$ be a complete Kähler manifold satisfying (2.1) and (2.2). On the parabolic end $E_{2}$, the function $f$ satisfies the gradient estimate

$$
|\nabla f|(x) \leq C \exp (2 m r(x))
$$

as $x \rightarrow \infty$ and $x \in E_{2}$ with $r(x)$ being the distance from $x$ to the fixed point $p \in M$.

Proof. Let $u$ be the harmonic function defined on $E_{2}$ obtained from the above construction. Observe that since the Ricci curvature is bounded from below, the gradient estimate of Cheng-Yau [2-1 (also see [1] implies that

$$
|\nabla(\log u)|^{2} \leq C
$$

on $E_{2} \backslash E_{2}\left(R_{0}+1\right)$. Integrating along a geodesic joining from $x \in$ $E_{2} \backslash E_{2}\left(R_{0}+1\right)$ to $\partial B_{p}\left(R_{0}+1\right) \cap E_{2}$, this implies that

$$
u(x) \leq C_{6} \exp (C r(x)) .
$$

Applying the gradient estimate again, this yields the estimate

$$
|\nabla u|(x) \leq C u(x) \leq C_{7} \exp (C r(x))
$$

for some constant $C_{7}>0$.

For $R>0$, let us denote

$$
s_{i}(R)=\sup _{x \in \partial B_{p}(R) \cap E_{2}}\left|\nabla u_{i}\right| .
$$

Since $u_{i}$ is harmonic, the Ricci curvature bound implies that $\left|\nabla u_{i}\right|$ satisfies the Bochner formula

$$
\Delta\left|\nabla u_{i}\right| \geq-2(m+1)\left|\nabla u_{i}\right| .
$$

If $x \in \partial B_{p}(R)$ such that $s_{i}(R)=\left|\nabla u_{i}\right|(x)$, then the mean value inequality of Li-Tam [1] 10 implies that

$$
\left|\nabla u_{i}\right|^{2}(x) V_{x}(1) \leq C \int_{B_{x}(1)}\left|\nabla u_{i}\right|^{2} .
$$


Combining with the volume lower bound (2.4), we have

$$
s_{i}^{2}(R) \leq C \exp (2 m R) \int_{B_{x}(1)}\left|\nabla u_{i}\right|^{2} .
$$

On the other hand, if we let $a=\inf _{B_{x}(1)} u_{i}$ and $b=\sup _{B_{x}(1)} u_{i}$, then

$$
\int_{B_{x}(1)}\left|\nabla u_{i}\right|^{2} \leq \int_{\Omega_{b} \backslash \Omega_{a}}\left|\nabla u_{i}\right|^{2},
$$

where $\Omega_{a}=\left\{x \mid u_{i}(x) \leq a\right\}$. Note that by the maximum principle, if $x \in E_{2} \backslash E_{2}\left(R_{0}+2\right)$ and for $i$ sufficiently large, then $0<a<b<c_{i}$ and the set $\Omega_{b} \backslash \Omega_{a}$ is bounded. Hence, the quantity on the right-hand side is finite. However, Stoke's theorem yields that

$$
\int_{\Omega_{b} \backslash \Omega_{a}}\left|\nabla u_{i}\right|^{2}=b \int_{\partial \Omega_{b}} \frac{\partial u_{i}}{\partial \nu}-a \int_{\partial \Omega_{a}} \frac{\partial u_{i}}{\partial \nu}
$$

where $\nu$ is the outward unit normal to the sets $\partial \Omega_{a}$ and $\partial \Omega_{b}$. On the other hand, we also have

$$
\begin{aligned}
0 & =\int_{\Omega_{a}} \Delta u_{i} \\
& =\int_{\partial \Omega_{a}} \frac{\partial u_{i}}{\partial \nu}-\int_{\partial B_{p}\left(R_{0}\right) \cap E_{2}} \frac{\partial u_{i}}{\partial \nu}
\end{aligned}
$$

for any $a>0$. Therefore, we conclude that

$$
\begin{aligned}
s_{i}^{2}(R) & \leq C \exp (2 m R)\left(\sup _{B_{x}(1)} u_{i}-\inf _{B_{x}(1)} u_{i}\right) \int_{\partial B_{p}\left(R_{0}\right) \cap E_{2}} \frac{\partial u_{i}}{\partial \nu} \\
& \leq C \exp (2 m R) \sup _{B_{x}(1)}\left|\nabla u_{i}\right| \int_{\partial B_{p}\left(R_{0}\right) \cap E_{2}} \frac{\partial u_{i}}{\partial \nu} \\
& \leq C \exp (2 m R) s_{i}(R+1) \int_{\partial B_{p}\left(R_{0}\right) \cap E_{2}} \frac{\partial u_{i}}{\partial \nu} .
\end{aligned}
$$

Setting

$$
A=\left(C \int_{\partial B_{p}\left(R_{0}\right) \cap E_{2}} \frac{\partial u_{i}}{\partial \nu}\right)^{\frac{1}{2}}
$$

we can rewrite the above inequality as

$$
s_{i}(R) \leq A \exp (m R) s_{i}^{\frac{1}{2}}(R+1) .
$$

Iterating this inequality $k$ times, we conclude that

$$
s_{i}(R) \leq A^{\sum_{j=0}^{k-1} 2^{-j}} \exp \left(\sum_{j=0}^{k-1} 2^{-j} m(R+j)\right) s_{i}^{2^{-k}}(R+k) .
$$


Letting $i \rightarrow \infty$, this implies that

$$
s(R) \leq A^{\sum_{j=0}^{k-1} 2^{-j}} \exp \left(\sum_{j=0}^{k-1} 2^{-j} m(R+j)\right) s^{2^{-k}}(R+k)
$$

where $s(R)=\sup _{\partial E_{2}(R)}|\nabla u|$. Note that since

$$
\begin{aligned}
\sum_{j=0}^{\infty} 2^{-j} & =2, \\
\exp \left(\sum_{j=0}^{\infty} 2^{-j} m(R+j)\right) & =\exp \left(\sum_{j=0}^{\infty} j 2^{-j}\right) \exp (2 m R) \\
& =C \exp (2 m R),
\end{aligned}
$$

and by $(2.7)$

$$
\begin{aligned}
\lim _{k \rightarrow \infty} s^{2^{-k}}(R+k) & \leq \lim _{k \rightarrow \infty} C_{7}^{2^{-k}} \exp \left(C(R+k) 2^{-k}\right) \\
& =1,
\end{aligned}
$$

after letting $k \rightarrow \infty$ in (2.8), we obtain

$$
\begin{aligned}
s(R) & \leq C \exp (2 m R) \\
& =C \exp (2 m R) .
\end{aligned}
$$

Integrating along geodesics, this gives the estimate

$$
u(x) \leq C \exp (2 m r(x))
$$

as $x \rightarrow \infty$ and $x \in E_{2}$. Since $f-u$ is bounded on $E_{2}$, the same upper bound is valid on $f$. Applying the gradient estimate (2.6) on $f$, we obtain the growth estimate as claimed.

q.e.d.

Corollary 2.4. Let $M$ be a complete Kähler manifold satisfying (2.1) and (2.2). There exists a constant $C>0$ such that the complex Hessian of $f$ satisfies the growth estimate

$$
\int_{B_{p}(R)}\left|f_{\alpha \bar{\beta}}\right| \leq C R
$$

for all $R \geq 1$.

Proof. Using the fact $f$ is harmonic and the Ricci curvature of $M$ has uniform lower bound, from the Bochner formula and a standard cut-off 
argument, we have

$$
\begin{aligned}
\int_{\left(B_{p}(R+2) \backslash B_{p}(R+1)\right) \backslash E_{2}}\left|f_{\alpha \bar{\beta}}\right|^{2} & \leq C \int_{\left(B_{p}(R+3) \backslash B_{p}(R)\right) \backslash E_{2}}|\nabla f|^{2} \\
& \leq C \exp (-2 m R),
\end{aligned}
$$

where we used (2.5) for the last inequality.

On the end $E_{2}$, Theorem 2.3 and the volume decay estimate in Corollary 2 imply that

$$
\begin{aligned}
\int_{\left(B_{p}(R+2) \backslash B_{p}(R+1)\right) \cap E_{2}}\left|f_{\alpha \bar{\beta}}\right|^{2} & \leq C \int_{\left(B_{p}(R+3) \backslash B_{p}(R)\right) \cap E_{2}}|\nabla f|^{2} \\
& \leq C \exp (2 m R) .
\end{aligned}
$$

Combining these two estimates, we conclude that

$$
\begin{aligned}
& \int_{B_{p}(R+2) \backslash B_{p}(R+1)}\left|f_{\alpha \bar{\beta}}\right| \\
& =\int_{\left(B_{p}(R+2) \backslash B_{p}(R+1)\right) \backslash E_{2}}\left|f_{\alpha \bar{\beta}}\right|+\int_{\left(B_{p}(R+2) \backslash B_{p}(R+1)\right) \cap E_{2}}\left|f_{\alpha \bar{\beta}}\right| \\
& \quad \leq\left(\int_{\left(B_{p}(R+2) \backslash B_{p}(R+1)\right) \backslash E_{2}}\left|f_{\alpha \bar{\beta}}\right|^{2}\right)^{\frac{1}{2}} V_{p}^{\frac{1}{2}}(R+2) \\
& \quad+\left(\int_{\left(B_{p}(R+2) \backslash B_{p}(R+1)\right) \cap E_{2}}\left|f_{\alpha \bar{\beta}}\right|^{2}\right)^{\frac{1}{2}}\left(V_{E_{2}}(R+2)-V_{E_{2}}(R+1)\right)^{\frac{1}{2}} \\
& \quad \leq C .
\end{aligned}
$$

The corollary now follows by iterating and summing over this estimate.

q.e.d.

\section{Infinite volume ends}

In this section, we will prove that for a broad class of Kähler manifolds, there are only one end with infinite volume. A version of this theorem was first proved in [i] i ] where the authors assumed a lower bound on the Ricci curvature. In the following theorem, we will present a version which a lower bound of the holomorphic bisectional curvature is assumed.

Theorem 3.1. Let $M$ be a complete Kähler manifold of complex dimension $m$. Suppose the holomorphic bisectional curvature of $M$ is 
bounded below by $B K_{M} \geq-1$ and the bottom of the spectrum $\lambda_{1}(M)$ of $M$ satisfies

$$
\lambda_{1}(M)^{\frac{3}{2}}+m \lambda_{1}(M)-m(m+1)>0 .
$$

Then, $M$ must have only one end with infinite volume. In particular, if

$$
\lambda_{1}(M) \geq m,
$$

then $M$ must have only one end with infinite volume.

Proof. Following the proof of Theorem 2.1 in [1] in $M$ has more than one infinite volume ends, then there exists a harmonic function $f$ with finite Dirichlet integral. It follows from Lemma 3.1 of $\left[\overline{8}_{i}\right]$ that it must be pluriharmonic. On the other hand, if we set $h=|\nabla f|$, then the Bochner formula for pluriharmonic function (see [i; $\left[\begin{array}{l}{[} \\ ]\end{array}\right]$ ) becomes

$$
\Delta h \geq-2(m+1) h+\frac{|\nabla h|^{2}}{h},
$$

since the assumptions on the holomorphic bisectional curvature imply that the Ricci curvature of $M$ is bounded by

$$
\operatorname{Ric}_{M} \geq-2(m+1) \text {. }
$$

If we let $g=h^{p}, 0<p<1$, then by an argument similar to (2.5) of

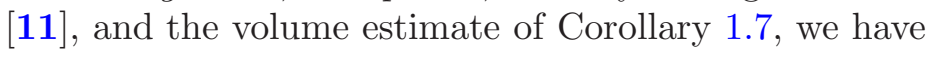

$$
\begin{aligned}
& \int_{B_{p}(2 R) \backslash B_{p}(R)} g^{2} \\
& \leq C R^{p}\left(\int_{R}^{2 R} \exp \left(-\frac{p}{1-p} 2 \sqrt{\lambda_{1}(M)} r\right) \exp (2 m r) d r\right)^{1-p} .
\end{aligned}
$$

Choosing $p$ to satisfy

$$
p \sqrt{\lambda_{1}(M)}=(1-p) m
$$

we conclude that

$$
\int_{B_{p}(R)} g^{2}=O(R)
$$

Moreover, since (3.1) implies that $g=h^{p}$ satisfies

$$
\Delta g \geq-2 p(m+1) g+\frac{|\nabla g|^{2}}{g}
$$

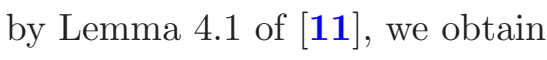

$$
\left(\lambda_{1}(M)-\frac{2 p(m+1)(1+\delta)}{1+2 \delta}\right) \int_{M} \phi^{2} g^{2} \leq\left(1+\frac{\delta^{2}}{1+2 \delta}\right) \int_{M}|\nabla \phi|^{2} g^{2}
$$

for all $\delta>0$. Now, if

$$
\lambda_{1}(M)>p(m+1)
$$


then there exists a sufficiently large $\delta$ such that

$$
\lambda_{1}(M)-\frac{2 p(m+1)(1+\delta)}{1+2 \delta}>0 .
$$

Arguing as in Theorem 4.2 of [1] 1 , we conclude that $g=0$ and $M$ has only one infinite volume end. However, condition (3.2) for $p$ asserts that

$$
p=\frac{m}{m+\sqrt{\lambda_{1}(M)}}
$$

hence, we need

$$
\lambda_{1}(M)\left(m+\sqrt{\lambda_{1}(M)}\right)-m(m+1)>0 .
$$

This proves the first part of the theorem.

Note that since the function

$$
q(x)=x^{3}+m x^{2}-m(m+1)
$$

is strictly increasing when $x>0$ with $q(0)<0$, (3.3) will be fulfilled as long as $\lambda_{1}(M)>x_{0}^{2}$, where $x_{0}>0$ is the positive solution to the cubic

$$
x^{3}+m x^{2}-m(m+1)=0 .
$$

The second part follows by observing that $q(\sqrt{m})=m(\sqrt{m}-1) \geq 0$. Hence, the assumption that $\lambda_{1}(M) \geq m$ implies that $\lambda_{1}(M)>x_{0}^{2}$ for $m>1$.

Following the argument in [1] ness theorem.

Theorem 3.2. Let $M$ be a complete Kähler manifold of complex dimension $m$. Let $x_{0}$ be the unique positive solution to the cubic

$$
x^{3}+m x^{2}-m(m+1)=0 .
$$

Suppose there exists a geodesic ball $B_{p}\left(R_{0}\right) \subset M$ such that $\lambda_{1}(M \backslash$ $\left.B_{p}\left(R_{0}\right)\right) \geq x_{0}^{2}+\epsilon$ for some $\epsilon>0$. Also assume that

$$
B K_{M} \geq-1
$$

on $M \backslash B_{p}\left(R_{0}\right)$. Then, $M$ must have finitely many ends with infinite volume. In particular, there exists a constant $C\left(m, R_{0}, \alpha, v, \epsilon\right)>0$ depending on the quantities $n, R_{0}, \epsilon, \alpha=\inf _{B_{p}\left(3 R_{0}\right)} \operatorname{Ric}_{M}$, and $v=$ $\inf _{x \in B_{p}\left(2 R_{0}\right)} V_{x}\left(R_{0}\right)$, such that the number of infinite volume ends of $M$ is at most $C$. 


\section{Finite volume ends}

To deal with finite volume ends, since the constructed harmonic function may not be pluriharmonic, we will utilize a Bochner type formula for the Laplacian of the length of the complex Hessian.

Lemma 4.1. Let $M$ be a Kähler manifold with complex dimension $m$. If $f$ is a harmonic function on $M$, then

$$
\Delta\left|f_{\alpha \bar{\beta}}\right|^{2} \geq-8 m \rho\left|f_{\alpha \bar{\beta}}\right|^{2}+\left.\left.\frac{m+1}{2 m}\left|f_{\alpha \bar{\beta}}\right|^{-2}|\nabla| f_{\alpha \bar{\beta}}\right|^{2}\right|^{2}
$$

where $f_{\alpha \bar{\beta}}$ denotes the complex Hessian of $f$ and $B K_{M}(x) \geq-\rho(x)$ is the pointwise lower bound of the holomorphic bisectional curvature of $M$.

Proof. Let $\left\{z^{1}, \cdots, z^{m}\right\}$ be complex normal coordinates at a point $z \in M$. The Hermitian metric can be written in the form

$$
d s^{2}=h_{\alpha \bar{\beta}} d z^{\alpha} \bar{z}^{\beta}
$$

where $1 \leq \alpha, \beta \leq m$. Using the Kähler condition

$$
\begin{aligned}
\frac{1}{4} \Delta\left|f_{\alpha \bar{\beta}}\right|^{2}= & \square\left|f_{\alpha \bar{\beta}}\right|^{2} \\
= & h^{\theta \bar{\eta}} \partial_{\theta} \partial_{\bar{\eta}}\left(h^{\alpha \bar{\gamma}} h^{\tau \bar{\beta}} f_{\alpha \bar{\beta}} f_{\tau \bar{\gamma}}\right) \\
= & h^{\theta \bar{\eta}}\left(\left(\partial_{\theta} \partial_{\bar{\eta}} h^{\alpha \bar{\gamma}}\right) h^{\tau \bar{\beta}} f_{\alpha \bar{\beta}} f_{\tau \bar{\gamma}}+h^{\alpha \bar{\gamma}}\left(\partial_{\theta} \partial_{\bar{\eta}} h^{\tau \bar{\beta}}\right) f_{\alpha \bar{\beta}} f_{\tau \bar{\beta}}\right. \\
& \quad+h^{\alpha \bar{\gamma}} h^{\tau \bar{\beta}}\left(\partial_{\theta} f_{\alpha \bar{\beta}}\right)\left(\partial_{\bar{\eta}} f_{\tau \bar{\gamma}}\right)+h^{\alpha \bar{\gamma}} h^{\tau \bar{\beta}}\left(\partial_{\bar{\eta}} f_{\alpha \bar{\beta}}\right)\left(\partial_{\theta} f_{\tau \bar{\gamma}}\right) \\
& \left.\quad+h^{\alpha \bar{\gamma}} h^{\tau \bar{\beta}}\left(\partial_{\theta} \partial_{\bar{\eta}} f_{\alpha \bar{\beta}}\right) f_{\tau \bar{\gamma}}+h^{\alpha \bar{\gamma}} h^{\tau \bar{\beta}} f_{\alpha \bar{\beta}}\left(\partial_{\theta} \partial_{\bar{\eta}} f_{\tau \bar{\gamma}}\right)\right)
\end{aligned}
$$

at the point $z$, where we have used the assumption that $\partial_{\theta} h^{\alpha \bar{\gamma}}(z)=0=$ $\partial_{\bar{\eta}} h_{\alpha \bar{\gamma}}(z)$. Using the assumption that $\Delta f=0$, we have

$$
\begin{aligned}
0 & =\partial_{\alpha} \partial_{\bar{\beta}}\left(h^{\theta \bar{\eta}} f_{\theta \bar{\eta}}\right) \\
& =\left(\partial_{\alpha} \partial_{\bar{\beta}} h^{\theta \bar{\eta}}\right) f_{\theta \bar{\eta}}+h^{\theta \bar{\eta}} \partial_{\alpha} \partial_{\bar{\beta}}\left(f_{\theta \bar{\eta}}\right) \\
& =\left(\partial_{\alpha} \partial_{\bar{\beta}} h^{\theta \bar{\eta}}\right) f_{\theta \bar{\eta}}+h^{\theta \bar{\eta}} \partial_{\theta} \partial_{\bar{\eta}}\left(f_{\alpha \bar{\beta}}\right) .
\end{aligned}
$$

The Kähler condition also implies that

$$
\partial_{\theta} \partial_{\bar{\eta}} h^{\alpha \bar{\gamma}}=R_{\bar{\eta}}^{\bar{\gamma}} \bar{\mu} \theta h^{\alpha \bar{\mu}}
$$


Substituting (4.2) and (4.3) this into (4.1) yields

$$
\begin{gathered}
\frac{1}{4} \Delta\left|f_{\alpha \bar{\beta}}\right|^{2}=h^{\theta \bar{\eta}} h^{\alpha \bar{\mu}} h^{\tau \bar{\beta}} R_{\bar{\eta}}^{\bar{\gamma}} \bar{\mu} \theta f_{\alpha \bar{\beta}} f_{\tau \bar{\gamma}} \\
+h^{\theta \bar{\eta}} h^{\alpha \bar{\gamma}} h^{\tau \bar{\mu}} R_{\bar{\eta}} \bar{\mu} \theta f_{\alpha \bar{\beta}} f_{\tau \bar{\gamma}}+2\left|\partial_{\theta} f_{\alpha \bar{\beta}}\right|^{2} \\
\quad-h^{\alpha \bar{\gamma}} h^{\tau \bar{\beta}} h^{\theta \bar{\delta}} R_{\bar{\beta}} \bar{\eta}_{\bar{\delta} \alpha} f_{\theta \bar{\eta}} f_{\tau \bar{\gamma}}-h^{\alpha \bar{\gamma}} h^{\tau \bar{\beta}} h^{\theta \bar{\delta}} R_{\bar{\gamma}}{ }_{\bar{\gamma}} f_{\theta \bar{\eta}} f_{\alpha \bar{\beta}} \\
=2 h^{\theta \bar{\eta}} h^{\alpha \bar{\mu}} h^{\tau \bar{\beta}} R_{\bar{\eta}} \bar{\gamma} \bar{\mu} \theta f_{\alpha \bar{\beta}} f_{\tau \bar{\gamma}}+2\left|\partial_{\theta} f_{\alpha \bar{\beta}}\right|^{2} \\
-2 h^{\alpha \bar{\gamma}} h^{\tau \bar{\beta}} h^{\theta \bar{\delta}} R_{\bar{\beta}}^{\bar{\eta}} \bar{\delta} \alpha f_{\theta \bar{\eta}} f_{\tau \bar{\gamma}} .
\end{gathered}
$$

At a fixed point $z \in M$, let us choose normal coordinates so that

$$
f_{\alpha \bar{\beta}}=\lambda_{\alpha} \delta_{\alpha \bar{\beta}}
$$

and

$$
h_{\alpha \bar{\beta}}=\delta_{\alpha \bar{\beta}} .
$$

This implies that

$$
2 h^{\theta \bar{\eta}} h^{\alpha \bar{\mu}} h^{\tau \bar{\beta}} R_{\bar{\eta}}^{\bar{\gamma}} \bar{\mu} \theta f_{\alpha \bar{\beta}} f_{\tau \bar{\gamma}}=2 R_{\bar{\eta}}{ }^{\bar{\alpha}}{ }_{\bar{\alpha} \eta} \lambda_{\alpha}^{2}
$$

and

$$
2 h^{\alpha \bar{\gamma}} h^{\tau \bar{\beta}} h^{\theta \bar{\delta}} R_{\bar{\beta}}^{\bar{\eta}} \bar{\delta} \alpha f_{\theta \bar{\eta}} f_{\tau \bar{\gamma}}=2 R_{\bar{\tau}}^{\bar{\theta}} \bar{\theta} \tau \lambda_{\theta} \lambda_{\tau} .
$$

Hence, the assumption on the bisectional curvature and two curvature terms in (4.4) combine to become

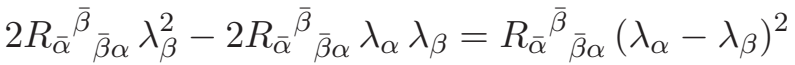

$$
\begin{aligned}
& \geq-\rho\left(\lambda_{\alpha}-\lambda_{\beta}\right)^{2} \text {. }
\end{aligned}
$$

However, $f$ is harmonic implies that

$$
\sum_{\alpha} \lambda_{\alpha}=0
$$

Hence,

$$
\begin{aligned}
2 R_{\bar{\alpha}}^{\bar{\beta}}{ }_{\bar{\beta} \alpha} \lambda_{\beta}^{2}-2 R_{\bar{\alpha}}^{\bar{\beta}}{ }_{\bar{\beta} \alpha} \lambda_{\alpha} \lambda_{\beta} & \geq-2 m \rho \sum_{\alpha} \lambda_{\alpha}^{2}-2 \rho \sum_{\alpha, \beta} \lambda_{\alpha} \lambda_{\beta} \\
& =-2 m \rho\left|f_{\alpha \bar{\beta}}\right|^{2} .
\end{aligned}
$$

Substituting this estimate into (4.4), we conclude that

$$
\frac{1}{4} \Delta\left|f_{\alpha \bar{\beta}}\right|^{2} \geq 2\left|\partial_{\theta} f_{\alpha \bar{\beta}}\right|^{2}-2 m \rho\left|f_{\alpha \bar{\beta}}\right|^{2}
$$


On the other hand, let us now consider the term

$$
\begin{aligned}
\left.\left.\frac{1}{4}|\nabla| f_{\alpha \bar{\beta}}\right|^{2}\right|^{2} & =h^{\theta \bar{\eta}} \partial_{\theta}\left|f_{\alpha \bar{\beta}}\right|^{2} \partial_{\bar{\eta}}\left|f_{\tau \bar{\gamma}}\right|^{2} \\
& =h^{\theta \bar{\eta}}\left(f_{\bar{\alpha} \beta} \partial_{\theta} f_{\alpha \bar{\beta}}+f_{\alpha \bar{\beta}} \partial_{\theta} f_{\bar{\alpha} \beta}\right)\left(f_{\bar{\tau} \gamma} \partial_{\bar{\eta}} f_{\tau \bar{\gamma}}+f_{\tau \bar{\gamma}} \partial_{\bar{\eta}} f_{\bar{\tau} \gamma}\right) .
\end{aligned}
$$

At the point $z \in M$, this can be written as

$$
\begin{aligned}
\left.\left.|\nabla| f_{\alpha \bar{\beta}}\right|^{2}\right|^{2} & =16 h^{\theta \bar{\eta}}\left(\lambda_{\alpha} \partial_{\theta} f_{\alpha \bar{\alpha}}\right)\left(\lambda_{\gamma} \partial_{\bar{\eta}} f_{\gamma \bar{\gamma}}\right) \\
& \leq 16\left|f_{\alpha \bar{\beta}}\right|^{2}\left|\partial_{\theta} f_{\alpha \bar{\alpha}}\right|^{2} .
\end{aligned}
$$

Also note that

$$
\begin{aligned}
\left|\partial_{\theta} f_{\alpha \bar{\beta}}\right|^{2} & =\left|\partial_{\theta} f_{\alpha \bar{\alpha}}\right|^{2}+\sum_{\bar{\beta} \neq \bar{\alpha}}\left|\partial_{\theta} f_{\alpha \bar{\beta}}\right|^{2} \\
& \geq\left|\partial_{\theta} f_{\alpha \bar{\alpha}}\right|^{2}+\sum_{\bar{\theta} \neq \bar{\alpha}}\left|\partial_{\theta} f_{\alpha \bar{\theta}}\right|^{2} \\
& =\left|\partial_{\theta} f_{\alpha \bar{\alpha}}\right|^{2}+\sum_{\bar{\theta} \neq \bar{\alpha}}\left|\partial_{\alpha} f_{\theta \bar{\theta}}\right|^{2} .
\end{aligned}
$$

Using the fact that $f$ is harmonic yields

$$
\begin{aligned}
(m-1) \sum_{\bar{\theta} \neq \bar{\alpha}}\left|\partial_{\alpha} f_{\theta \bar{\theta}}\right|^{2} & \geq\left|\sum_{\bar{\theta} \neq \bar{\alpha}} \partial_{\alpha} f_{\theta \bar{\theta}}\right|^{2} \\
& =\left|\partial_{\alpha} f_{\alpha \bar{\alpha}}\right|^{2},
\end{aligned}
$$

hence,

$$
m \sum_{\bar{\theta} \neq \bar{\alpha}}\left|\partial_{\alpha} f_{\theta \bar{\theta}}\right|^{2} \geq\left|\partial_{\theta} f_{\alpha \bar{\alpha}}\right|^{2} .
$$

Combining this with (4.7), we concude that

$$
\left|\partial_{\theta} f_{\alpha \bar{\beta}}\right|^{2} \geq \frac{m+1}{m}\left|\partial_{\theta} f_{\alpha \bar{\alpha}}\right|^{2} .
$$

Substituting this estimate into (4.6) gives

$$
\left.\left.|\nabla| f_{\alpha \bar{\beta}}\right|^{2}\right|^{2} \leq \frac{16 m}{m+1}\left|f_{\alpha \bar{\beta}}\right|^{2}\left|\partial_{\theta} f_{\alpha \bar{\beta}}\right|^{2} .
$$

The lemma follows by combining this with (4.5).

We now restrict our attention to the case $m=2$.

Lemma 4.2. Let $M$ be a complete Kähler manifold of complex dimension 2. Suppose the holomorphic bisectional curvature of $M$ is bounded from below by $B K_{M} \geq-1$ and the bottom of the spectrum of 
the Laplacian for functions is bounded by $\lambda_{1}(M) \geq 4$. If $f$ is a harmonic function on $M$ whose complex Hessian satisfies the growth estimate

$$
\int_{B_{x}(r)}\left|f_{\alpha \bar{\beta}}\right| \leq o\left(r^{2}\right)
$$

as $r \rightarrow \infty$, then $f$ must either be pluriharmonic, or the function

$$
g=\left|f_{\alpha \bar{\beta}}\right|^{\frac{1}{2}}
$$

must be positive and satisfy the equation

$$
\Delta g=-4 g \text {. }
$$

Proof. Let $g=\left|f_{\alpha \bar{\beta}}\right|^{\frac{1}{2}}$. Lemma $\overline{4} \overline{1} \overline{1}_{1}^{\prime}$ asserts that

$$
\Delta g \geq-2 m g-\frac{m-2}{m} g^{-1}|\nabla g|^{2} .
$$

When $m=2$, this becomes

$$
\Delta g \geq-4 g .
$$

For a non-negative compactly supported function $\phi$ defined on $M$, applying the assumption on the spectrum, then

$$
\begin{aligned}
4 \int_{M}(\phi g)^{2} & \leq \int_{M}|\nabla(\phi g)|^{2} \\
& =\int_{M}|\nabla \phi|^{2} g^{2}-\int_{M} \phi^{2} g \Delta g .
\end{aligned}
$$

So, we have

$$
\int_{M} \phi^{2} g(\Delta g+4 g) \leq \int_{M}|\nabla \phi|^{2} g^{2} .
$$

If we choose the function

$$
\phi(x)=\left\{\begin{array}{cll}
1 & \text { on } & B_{p}(R) \\
\frac{2 R-r(x)}{R} & \text { on } & B_{p}(2 R) \backslash B_{p}(R) \\
0 & \text { on } & M \backslash B_{p}(2 R),
\end{array}\right.
$$

then

$$
\int_{M}|\nabla \phi|^{2} g^{2} \leq R^{-2} \int_{B_{p}(2 R) \backslash B_{p}(R)} g^{2} .
$$

Since the right-hand side tends to 0 as $R \rightarrow \infty$ due to the growth assumption on $g$, we conclude that all the inequalities used in the proof and Lemma 
If $f_{\alpha \bar{\beta}}$ is identitally 0 , then this implies that $f$ is pluriharmonic. Otherwise, $g=\left|f_{\alpha \bar{\beta}}\right|^{\frac{1}{2}}$ must satisfy

$$
\Delta g=-4 g \text {. }
$$

We now claim that $g>0$. Indeed, if $g=0$ at some point, then by regularity of the equation (4.11), g must change sign. However, since $g \geq 0$, this is impossible.

Since inequality (4.8) becomes equality, we have

$$
\partial_{\alpha} f_{\theta \bar{\theta}}=\partial_{\alpha} f_{\eta \bar{\eta}}
$$

for all $\theta, \eta \neq \alpha$. In particular, this implies that

$$
\partial_{\alpha} f_{\alpha \bar{\alpha}}=(m-1) \partial_{\alpha} f_{\theta \bar{\theta}}
$$

for all $\theta \neq \alpha$. Also, the fact that inequality (4.7) becomes equality implies that

$$
\partial_{\theta} f_{\alpha \bar{\beta}}=0
$$

for all $\theta \neq \beta$ and $\beta \neq \alpha$.

q.e.d.

Theorem 4.3. Let $M^{2}$ be a complete Kähler manifold of complex dimension 2. Suppose the holomorphic bisectional curvature of $M$ is bounded by $B K_{M} \geq-1$. If the bottom of the spectrum of the Laplacian, $\lambda_{1}(M)$, is bounded from below by $\lambda_{1}(M) \geq 4$, then $M$ must have at most four ends. Moreover, exactly one of its ends must have infinite volume, while the rest of the ends have finite volume.

Proof. As discussed earlier, the assumption $\lambda_{1}(M) \geq 4$ implies that $M$ must have exponential volume growth. In particular, one of the ends of $M$ must have infinite volume. Combining with Theorem 1 , we see that $M$ has exactly one infinite volume end $E_{1}$.

Let us now assume that $M$ has at least three ends. By the above discussion, other than $E_{1}$, all the other ends must have finite volume. For each finite volume end $E_{2}$, following the construction in [9], and [1] properties:

(1) $\sup _{\partial B_{p}(R) \cap E_{2}} f(x) \rightarrow \infty$ as $R \rightarrow \infty$;

(2) $\inf _{\partial B_{p}(R) \cap E_{1}} f(x) \rightarrow 0$ as $R \rightarrow \infty$; and

(3) $f$ is bounded on all other ends.

Moreover, it also follows that $f$ has finite Dirichlet integral on $E_{1}$. In fact, we also derive the growth estimate in Corollary 2.4 that

$$
\int_{B_{p}(R)}\left|f_{\alpha \bar{\beta}}\right| \leq C R .
$$


Applying Lemma 4.2, , we conclude that either $f$ is pluriharmonic, or that $g=\left|f_{\alpha \bar{\beta}}\right|^{\frac{1}{2}}$ is positive satisfying the equation

$$
\Delta g=-4 g \text {. }
$$

If $f$ is pluriharmonic, then using the argument in Theorem 5.1 of [i] or Theorem 3.1, we conclude that this is impossible. Hence, $g$ must satisfy (4.12). Since there are more than one finite volume end, we can find at least two linearly independent harmonic functions $f$ and $\tilde{f}$, constructed using two small ends $E_{2}$ and $E_{3}$, such that

$$
g=\left|f_{\alpha \bar{\beta}}\right|^{\frac{1}{2}}
$$

and

$$
\tilde{g}=\left|\tilde{f}_{\alpha \bar{\beta}}\right|^{\frac{1}{2}}
$$

both satisfy (4.12). If $g$ is not a scalar multiple of $\tilde{g}$, then we can find a linear combination $G=a g+b \tilde{g}$ such that $G$ must change sign. Moreover, $G$ also satisfies

$$
\Delta G=-4 G
$$

The function $|G|$ will then satisfy

$$
\Delta|G| \geq-4|G|
$$

in the weak sense and must vanish somewhere. However, since $g$ and $\tilde{g}$ have $L^{2}$ norms satisfying the growth condition

$$
\int_{B_{p}(R)} g^{2} \leq C R
$$

and

$$
\int_{B_{p}(R)} \tilde{g}^{2} \leq C R
$$

the function $|G|$ will also satisfy the growth condition

$$
\int_{B_{p}(R)}|G|^{2} \leq C R
$$

Applying the cut-off argument on $|G|$ as in the proof of Lemma conclude that

$$
\Delta|G|=-4|G|
$$

The regularity argument of Lemma implies that this impossible. Hence, $g$ must be a scalar multiple of $\tilde{g}$. In particular, after a rescaling of $\tilde{f}$, we may assume that $g=\tilde{g}$.

Let us now choose a unitary frame $\left\{e_{1}, e_{2}, e_{\overline{1}}, e_{\overline{2}}\right\}$ such that

$$
\left(f_{\alpha \bar{\beta}}\right)=\left(\begin{array}{cc}
\mu & 0 \\
0 & -\mu
\end{array}\right) .
$$


Since

$$
\begin{aligned}
g^{4} & =\left|f_{\alpha \bar{\beta}}\right|^{2} \\
& =2 \mu^{2}
\end{aligned}
$$

is positive, the unitary frame is, in fact, globally defined. With respect to this frame, the complex Hessian of $\tilde{f}$ can be written as

$$
\left(\tilde{f}_{\alpha \bar{\beta}}\right)=\left(\begin{array}{cc}
\tilde{\mu} & \nu \\
\bar{\nu} & -\mu
\end{array}\right) .
$$

Since

$$
\begin{aligned}
g^{4} & =\left|\tilde{f}_{\alpha \bar{\beta}}\right|^{2} \\
& =2 \tilde{\mu}^{2}+2|\nu|^{2}
\end{aligned}
$$

we conclude that

$$
\mu^{2}=\tilde{\mu}^{2}+|\nu|^{2} .
$$

On the other hand, for any $0 \leq t \leq 1$, the function

$$
h=t f+(1-t) \tilde{f}
$$

is harmonic. Moreover, the above argument implies that there is a constant $\alpha>0$, depending only on $t$, such that

$$
\left|h_{\alpha \bar{\beta}}\right|^{2}=\alpha g^{2} \text {. }
$$

On the other hand, since

$$
\left(h_{\alpha \bar{\beta}}\right)=\left(\begin{array}{cc}
t \mu+(1-t) \tilde{\mu} & (1-t) \nu \\
(1-t) \bar{\nu} & -t \mu-(1-t) \tilde{\mu}
\end{array}\right)
$$

we have

$$
2 \alpha \mu^{2}=2(t \mu+(1-t) \tilde{\mu})^{2}+2(1-t)^{2}|\nu|^{2} .
$$

Using the identity (4.13), this implies that

$$
(\alpha-1) \mu=2 t(1-t) \tilde{\mu} .
$$

However, since

$$
\frac{\alpha-1}{2 t(t-1)}
$$

is independent on the point in $M$ and

$$
\frac{\tilde{\mu}}{\mu}
$$

is independent on $t$, we conclude that

$$
\tilde{\mu}=\beta \mu
$$


for some constant

$$
\beta=\frac{\alpha-1}{2 t(t-1)}
$$

Using (4.13) again, we deduce that

$$
\left(\tilde{f}_{\alpha \bar{\beta}}\right)=\left(\begin{array}{cc}
\beta \mu & \sqrt{1-\beta^{2}} \mu e^{i \theta} \\
\sqrt{1-\beta^{2}} \mu e^{-i \theta} & -\beta \mu
\end{array}\right)
$$

for some constant $0 \leq \theta<2 \pi$.

Note that if $\beta=1,-1$, then either $f-\tilde{f}$ or $f+\tilde{f}$ will be a nonconstant pluriharmonic function and the argument in [i1 i. $]$ will give a contradiction. Hence, $-1<\beta<1$ because of (4.13). In the event if $\beta \neq 0$, then the linear combination

$$
\frac{\tilde{f}-\beta f}{\sqrt{1-\beta^{2}}}
$$

will have complex Hessian of the form

$$
\left(\begin{array}{cc}
0 & \mu e^{-i \theta} \\
\mu e^{-i \theta} & 0
\end{array}\right)
$$

In any case, by possibly multiplying by -1 , we may then assume

$$
\left(\tilde{f}_{\alpha \bar{\beta}}\right)=\left(\begin{array}{cc}
0 & \mu e^{-i \theta} \\
\mu e^{-i \theta} & 0
\end{array}\right),
$$

for some constant $0 \leq \theta<\pi$.

Now, let us assume that $M$ has at least 5 ends. Since exactly one end has infinite volume, there must be at least 4 finite volume ends. Each of the ends with finite volume will produce a positive harmonic function as discussed above. Let us denote these functions by $\left\{f_{i}\right\}_{i=1}^{4}$. Using $f_{1}$ to play the role of $f$ and each $f_{j}$ playing the role of $\tilde{f}$, we conclude that their complex Hessians are of the form

$$
\left(\left(f_{1}\right)_{\alpha \bar{\beta}}\right)=\left(\begin{array}{cc}
\mu & 0 \\
0 & -\mu
\end{array}\right)
$$

and

$$
\left(\left(f_{j}\right)_{\alpha \bar{\beta}}\right)=\left(\begin{array}{cc}
0 & \mu e^{i \theta_{j}} \\
\mu e^{-i \theta_{j}} & 0
\end{array}\right)
$$

for $2 \leq j \leq 4$, where $0 \leq \theta_{j}<\pi$. Obviously, by taking linear combinations of $\left\{f_{j}\right\}_{j=2}^{4}$, we may arrange an $\tilde{f}$ in the subspace spanned by the $\left\{f_{j}\right\}$ such that its complex Hessian is identically 0. Hence, $\tilde{f}$ is a non-constant pluriharmonic function, which gives a contradiction. This implies that $M$ cannot have more than 4 ends.

q.e.d. 


\section{Acknowledgements}

The first author was partially supported by NSF grants DMS-0202508 and DMS-0112416. The second author was partially supported by NSF grant DMS-0072181.

\section{References}

[1] S.Y. Cheng, Eigenvalue comparison_theorems and its geometric applications, Math. Z. 143 (1975) 289-297, MR 10378001, Zbl 0329.53035'.

[2] S.Y. Cheng \& S.-T. Yau, Differential equations on Riemannian manifolds and their qeometric applications, Comm. Pure Appl. Math. 28 (1975) 333-354, MR 0385749, Zbl

[3] M. Cai \& G.J. Galloway, Boundaries of zero scalar curvature in the $A \underline{D} S / C F T$ correspondence, Adv. Theor. Math. Phys. 3 (1999) 1769-1783, MR 1812136, $\mathrm{Zbl}, 0978.53084$

[4] H.-D. Cao \& L. Ni, Matrix Li-Yau-Hamilton estimates for the heat equation on Kähler manifolds, to appear in Math. Ann..

[5] R.E. Greene \& H. Wu, On Kähler manifolds of positive bisectional curvature and a theorem of Hartogs, Abh. Math. Sem. Univ. Hamburg 47 (1978) 171-185, MR 0499318

[6] J. Lee, The spectrum of an asymptotically_hyperbolic Einstein manifold, Comm. Anal. Geom. 3 (1995) 253-271, MR 1362652, Zbl 0934.58029.

[7] P. Li, Lecture Notes on Geometric Analysis, Lecture Notes Series, 6, Research Institute of Mathematics and Global Analysis Research Center, Seoul National University, Seoul, 1993, MR 1320504, Zbl 0822.58001.

[8] P. Li, Curvature and function theory on Riemannian manifolds, in 'Survey in Differential Geometry "In Honor of Atiyah, Bott, Hirzebruch, and Singer",' International Press, Cambridge VII (2000) 71-111, MR 1919432.

[9] P. Li \& L.F. Tam, Harmonic functions and the structure of complete manifolds,

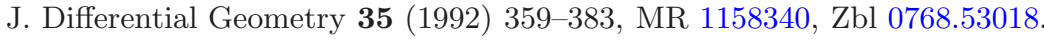

[10] P. Li \& L.F. Tam, The heat equation and harmonic maps_of complete manifolds, Invent. Math. 105 (1991) 1-46, MR 1109619, Zbl 0748.58006.

[11] P. Li \& J. Wang, Complete manifold___with positive spectrum, J. Differential Geometry 58 (2001) 501-534, MR 1906784, Zbl 1032.58016.

[12] P. Li \& J. Wang, Complete manifolds_with positive spectrum, II, J. Differential Geometry 62 (2002) 143-162, MR 1987380.

[13] P. Li \& S.-T. Yau, On the Parabolic Kernel of the Schrödinger Operator, Acta Math. 156 (1986) 153-201, MR ,0834612, Zbl,

[14] D. Sullivan, Related aspects of positivity in Riemannian geometry, J. Differential Geometry 25 (1987) 327-351, MR ,0882827, Zbl 0615.53029.

[15] C.J. Sung, L.F. Tam, \& J. Wang, Spaces of harmonic functions, J. London Math. Soc. 61 (2000) 789-806, MR '1766105, Zbl 0963.31004.

[16] X. Wang, On conformally compact Einstein manifolds, Math. Res. Let. 8 (2001) 671-688, MR 1879811. 
[17] E. Witten \& S.-T. Yau, Connectedness of the boundary in the AdS $\underline{S}-\underline{C} F$ correspondence, Adv. Theor. Math. Phys. 3 (1999) 1635-1655, MR 1812133, Zbl

Department of Mathematics

University of CALIFornia

IRVINE, CA 92697-3875

E-mail address: pli@math.uci.edu

School of Mathematics

UniVERSity OF MinNESOTA

MinNeAPOLIS, MN 55455

E-mail address: jiaping@math.umn.edu 